\title{
Dominant and recessive imprinting of exosomes from parent cells
}

\author{
Yangxin Li, Jin Zhou, Yao-Hua Song and Xi-Yong Yu
}

We read with great interest the Review by Boulanger et al. (Extracellular vesicles in coronary artery disease. Nat. Rev. Cardiol. 14, 259-272; 2017) ${ }^{1}$. The authors discuss the role of extracellular vesicles (including exosomes) as transporters of biological information between cells and tissues. Specifically, this Review provides an update on how exosomes released by stem cells might improve cardiac function after myocardial infarction. New studies suggest that many of the beneficial effects of exosomebased therapies are mediated by microRNAs ${ }^{2}$. In light of new exciting developments in exosome research, we wish to highlight some important additional points on how exosome functions are influenced by the imprinting of parent cells.

A study published in 2017 shows that exosomes derived from human $\mathrm{CD}_{3} 4^{+}$stem cells improved angiogenesis and motor function in mouse ischaemic limb, mimicking the effect of the parent cells, and that these effects were mediated by transferring the microRNA miR126-3p to endothelial cells ${ }^{3}$. By contrast, exosomes derived from $\mathrm{CD} 34^{+}-$ cell-depleted parent cells were ineffective ${ }^{3}$. These results suggest that $\mathrm{CD} 34^{+}$stem cells contain unique molecules that are delivered specifically to endothelial cells by exosomes. Another study published in 2017 demonstrates that the therapeutic effect of exosomes derived from human paediatric cardiac progenitor cells is influenced by both donor age and oxygen levels of the cell cultures ${ }^{2}$. These studies indicate that both the content and function of exosomes are determined by dominant imprinting of parent cells, which is regulated by the microenvironment surrounding the cells.

Moreover, exosomes also contain some mRNAs and microRNAs that are not functional in parent cells, but can be functional in recipient cells ${ }^{4}$. Similarly, a study in mice showed that exosomes derived from dendritic cells carry major histocompatibility complex class I and T cell co-stimulatory molecules that prime specific $\mathrm{T}$ cells to suppress the growth of tumours ${ }^{5}$. In some cases, exosomes can transfer antigens from tumours to dendritic cells, which in turn initiate a cytotoxic $T$ cell-dependent immune response against tumour cells ${ }^{6}$. Therefore, exploring how recessive imprinting from parent cells might influence the therapeutic effects of exosomes is worthwhile.

In summary, emerging evidence demonstrates that exosomes not only mimic the effects of their parent cells, but also have great potential to overcome limitations associated with cell therapies, such as poor engraftment and survival under a hostile ischaemic microenvironment ${ }^{7,8}$. However, the molecular mechanisms of exosome dynamic generation, transport, and uptake remain poorly understood. Exploration of how dominant and recessive imprinting influence the molecular signature of exosomes and identification of cell typespecific receptors will enable targeted delivery of exosomes ${ }^{9}$. In this context, the Review by Boulanger et al. ${ }^{1}$ provides new insights into exosome-based therapies for cardiovascular diseases.
Yangxin Li is at the Institute for Cardiovascular Science and Department of Cardiovascular Surgery, First Affiliated Hospital of Soochow University, Suzhou, Jiangsu 215123, China.

Jin Zhou is at the Department of General Surgery, The First Affiliated Hospital of Soochow University, Suzhou, Jiangsu 215123, China.

Yao-Hua Song is at the Cyrus Tang Hematology Center, Collaborative Innovation Center of Hematology, Soochow University, Suzhou, Jiangsu 215123, China.

$\mathrm{Xi}$-Yong Yu is at the Key Laboratory of Molecular Clinical Pharmacology and Fifth Affiliated Hospital, Guangzhou Medical University, Guangzhou, Guangdong 511436, China.

Correspondence to Y.L. yangxinli@yahoo.com doi: $10.1038 /$ nrcardio. 2017.93 Published online 15 Jun 2017

1. Boulanger, C. M., Loyer, X., Rautou, P. E. \& Amabile, N. Extracellular vesicles in coronary artery disease. Nat. Rev. Cardiol. 14, 259-272 (2017).

2. Agarwal, U. et al. Experimental, systems, and computational approaches to understanding the microRNA-mediated reparative potential of cardiac progenitor cell-derived exosomes from pediatric patients. Circ. Res. 120, 701-712 (2017).

3. Mathiyalagan, P. et al. Angiogenic mechanisms of human CD34 + stem cell exosomes in the repair of ischemic hindlimb. Circ. Res. 120, 1466-1476 (2017).

4. Boon, R. A. \& Vickers, K. C. Intercellular transport of microRNAs. Arterioscler. Thromb. Vasc. Biol. 33 , 186-192 (2013).

5. Zitvogel, L. et al. Eradication of established murine tumors using a novel cell-free vaccine: dendritic cell-derived exosomes. Nat. Med. 4, 594-600 (1998).

6. Wolfers, J. et al. Tumor-derived exosomes are a source of shared tumor rejection antigens for CTL cross-priming. Nat. Med. 7, 297-303 (2001).

7. Khan, M. et al. Embryonic stem cell-derived exosomes promote endogenous repair mechanisms and enhance cardiac function following myocardial infarction. Circ. Res. 117, 52-64 (2015).

8. Sahoo, S. \& Losordo, D. W. Exosomes and cardiac repair after myocardial infarction. Circ. Res. 114 333-344 (2014).

9. Christianson, H. C., Svensson, K. J., van Kuppevelt, T. H., Li, J. P. \& Belting, M. Cancer cell exosomes depend on cell-surface heparan sulfate proteoglycans for their internalization and functional activity. Proc. Natl Acad. Sci. USA 110, 17380-17385 (2013).

\section{Acknowledgements}

Y.L. and J.Z. contributed equally to this work. The authors were supported by Jiangsu Province Key Scientific and Technological Project (BE2016669), National Natural Science Foundation of China (81670358), Suzhou Science and Technology Project (SS201665), Priority Academic Program Development of Jiangsu Higher Education Institutions (PAPD), and Jiangsu Province's Key Discipline/Laboratory of Medicine (XK201118).

Competing interests statement

The authors declare no competing interests. 